\title{
Direct conversion of polyconjugated compounds into their corresponding carboxylic acids by Acetobacter aceti
}

\author{
Elena Pini ${ }^{\mathrm{a}, *}$, Vittorio Bertacche ${ }^{\mathrm{a}}$, Francesco Molinari ${ }^{\mathrm{b}}$, Diego Romano ${ }^{\mathrm{b}}$, Raffaella Gandolfi ${ }^{\mathrm{a}}$ \\ a Istituto di Chimica Organica 'A. Marchesini', Università degli Studi, Via Venezian 21, 20133 Milano, Italy \\ ${ }^{\mathrm{b}}$ Dipartimento di Scienze e Tecnologie Alimentari e Microbiologiche, Università degli Studi, Via Mangiagalli 25, 20133, Milano, Italy
}

\section{A R T I C L E I N F O}

\section{Article history:}

Received 30 April 2008

Received in revised form 13 June 2008

Accepted 3 July 2008

Available online $\mathrm{xxx}$

\section{Keywords:}

Polyconjugated compounds

Oxidation

Acetobacter aceti

\begin{abstract}
A B S T R A C T
The conversion of polyconjugated aldehydes or alcohols into their corresponding acids was carried out using Acetobacter aceti. The analytical results were compared with those of the acids chemically obtained using a Horner-Wittig reaction.
\end{abstract}

(c) 2008 Elsevier Ltd. All rights reserved.

\section{Introduction}

In recent years, the interest in polyconjugated systems has increased due to the antioxidant properties of these molecules. ${ }^{1-3}$ Polyunsaturated aldehydes and alcohols have been evaluated in a number of biochemical and pharmacological studies, ${ }^{4-6}$ which have identified their potential role as antioxidant and antiproliferative molecules.

Polyconjugated aldehydes were obtained following Kuhn ${ }^{7}$ and Blout $^{8}$ procedures by self-condensation of crotonaldehyde or by condensation between crotonaldehyde and acetaldehyde using piperidinium acetate as catalyst. The compounds obtained were subsequently reduced by $\mathrm{NaBH}_{4}$ giving the corresponding alcohols.

The aim of this work was the direct conversion of compounds $\mathbf{1}$ and $\mathbf{2}$ into the corresponding carboxylic acids $\mathbf{3}$ (Scheme 1). In the literature it is known that the conventional methods such as permanganate ${ }^{9}$ or Jones reagent ${ }^{10}$ could not be applied to $\alpha, \beta$-unsaturated compounds since double bonds are affected under these conditions. Balkrishna et al. ${ }^{11}$ suggested the use of sodium chlorite, but this method gave double bond oxidation with these molecules as well.

Nevertheless, several methods ${ }^{12-15}$ are available for the synthesis of $\alpha, \beta$-unsaturated carboxylic acids, but these reactions have low yields and did not allow the direct oxidation.

\footnotetext{
* Corresponding author. Tel.: +392 50314606; fax: +39250314615.

E-mail address: elena.pini@unimi.it (E. Pini).
}

To overcome these synthetic problems, microbial cells endowed with dehydrogenases or oxidases able to perform the selective oxidation of aldehydes and/or alcohols under mild conditions can be employed. ${ }^{16}$ Acetic acid bacteria are known to be particularly suited for carrying out these oxidations also on industrial scale. ${ }^{17,18}$

In this work, we have investigated the oxidation of different polyunsaturated aldehydes and alcohols catalysed by Acetobacter aceti MIM2000/28, a microorganism already used for a number of selective bio-oxidations. ${ }^{19,20}$

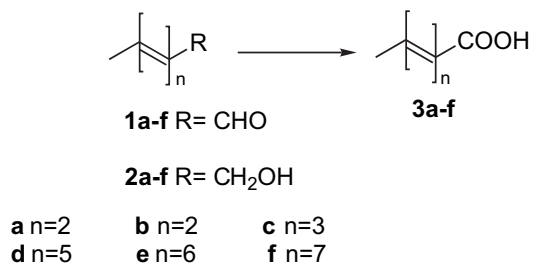

Scheme 1. Direct oxidation of polyconjugated aldehydes and alcohols.

\section{Results and discussion}

The direct chemical oxidation of polyconjugated aldehydes (1a-f, Scheme 1) with conventional reagents, such as potassium permanganate, Jones reagent and the Balkrishna method was unsuccessful.

The polyunsaturated carboxylic acids (3a-f) were obtained using an indirect method. The ethyl esters obtained by the 
Horner-Wittig reaction carried out between the all trans aldehydes and the ylide from triethyl phosphonoacetate were hydrolysed with methanolic potassium hydroxide ${ }^{21}$ (Scheme 2 ) leading to the carboxylic acids. In this case, the reaction product is a molecule with $n+1$ unsaturation compared with the starting aldehyde; the olefinic systems kept all trans configuration but the final yields were low due to the number of steps involved. All the acids obtained following this procedure were isolated and characterised by ${ }^{1} \mathrm{H}$ and ${ }^{13} \mathrm{C}$ NMR spectroscopies, two-dimensional analyses (COSY and HETCOR), infrared spectroscopy and mass spectrometry.

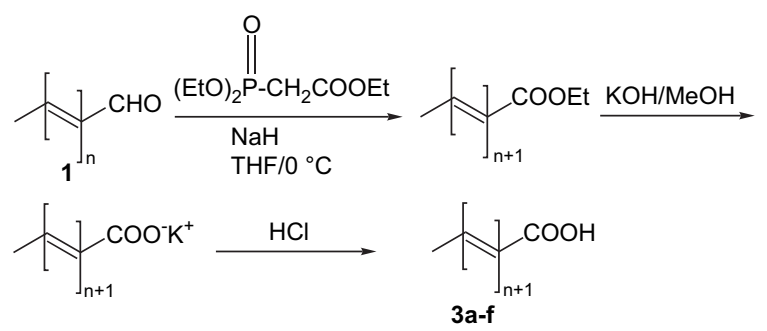

Scheme 2. Synthesis of polyunsaturated acids $\mathbf{3}$ by Horner-Wittig reaction.

A. aceti MIM2000/28 was used for the oxidation of the polyunsaturated aldehydes furnishing good yields compared with the ones obtained by chemical means (Table 1 ). The molar conversion decreased from 2,4-hexadienal 1a to 2,4,6,8,10-dodecapentaenal 1d; no reaction was observed with aldehydes having longer chain lengths (1e and $\mathbf{1 f}$ ). This trend can be due to the lower solubility in water of the substrates with higher chain length and/or to the increased toxicity of the substrates towards the enzymatic systems. The biocatalytic oxidation of aldehydes $1 \mathbf{c}$ and $\mathbf{1 d}$ reached maximum conversion after 24 and $48 \mathrm{~h}$, respectively; no change of substrate/product composition was observed at prolonged times. The time-course of the oxidation of 1d is reported in Figure 1.

The study was then extended to alcohols $\mathbf{2 a - f}$, obtained by reduction of the corresponding aldehydes with $\mathrm{NaBH}_{4}$; alcohols are generally more soluble in water, more stable and tend to be less toxic towards the dehydrogenases involved in these reactions. The results are summarised in Table 2.

The oxidation of the different polyconjugated alcohols catalysed by $A$. aceti occurred with no detectable aldehyde accumulation, indicating that the second step of the oxidation (aldehyde into acid) is faster than the first one (alcohol into aldehyde). The occurrence of different membrane-bound dehydrogenases in acetic acid bacteria has been exhaustively studied; normally aldehyde dehydrogenases are more active than alcohol dehydrogenases so that aldehydes (potentially toxic to many enzymes) are not accumulated. ${ }^{20,22}$

The conversion of these substrates was higher than the ones observed starting from the aldehydes. The time-course of the oxidation of $\mathbf{2 d}$ is reported in Figure 2.

Table 1

Molar conversion of aldehydes $\mathbf{1}$ into acids $\mathbf{3}$ obtained by chemical and biocatalytic methods

\begin{tabular}{llll}
\hline Acid & Chemical yield (\%) & Biocatalytic yield (\%) & $\begin{array}{l}\text { Biocatalytic time } \\
\text { conversion (h) }\end{array}$ \\
\hline 3a & 10 & 86 & 2 \\
3b & 23 & 89 & 3 \\
3c & 51 & $60^{\text {a }}$ & 24 \\
3d & 20 & $65^{\mathrm{a}}$ & 48 \\
3e & 10 & - & - \\
3f & 10 & - & - \\
\hline
\end{tabular}

a The resuspended cells' concentration was six times the concentrate compared with cell cultures. In the other cases the ratio was $2: 1$.

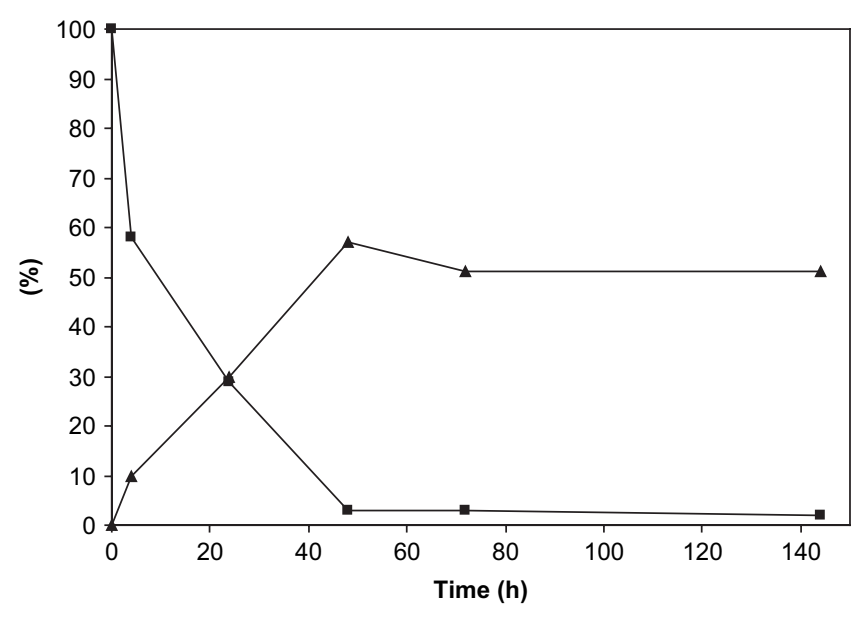

Figure 1. Time-course of the biotransformation of 2,4,6,8,10-dodecapentaenal 1d catalysed by A. aceti MIM2000/28. ם, aldehyde; $\boldsymbol{\Lambda}$, acid.

The acid yields lowered by increasing the chain length of the substrate, as observed when aldehydes were used as substrate.

\section{Conclusion}

The use of a biocatalytic approach for the direct oxidation of polyconjugated aldehydes seems to be a promising and possibly general method to furnish acids in good yields. The analytical profile of the biotransformation products is comparable to the profile of the chemically synthesised acids; moreover, the ${ }^{1} \mathrm{H}$ NMR spectroscopic analyses confirm the all trans configuration of double bonds.

\section{Experimental section}

\subsection{Materials and methods}

The ${ }^{1} \mathrm{H}$ and ${ }^{13} \mathrm{C}$ NMR spectra and two-dimensional analyses (COSY and HETCOR) were carried out on a Varian Gemini 200 operating at $200 \mathrm{MHz}$. Chemical shifts were expressed as parts per million $(\delta)$. Samples of about $5 \mathrm{mg}$ were dissolved in $0.7 \mathrm{~mL}$ of DMSO.

HPLC/DAD analyses were performed using a Perkin-Elmer Series 200 DAD equipped with a manual injector Rheodyne 7125 (loop $20 \mu \mathrm{L}$ ), an online degasser and a column block heater. The column employed was a Purosphere STAR RP 18-e (Merck, MA, USA), $150 \times 4.6 \mathrm{~mm}$ i.d. ( $5 \mu \mathrm{m}$ particle size); mobile phase was methanol with $0.1 \%$ acetic acid $(\mathrm{v} / \mathrm{v})$ eluted at $0.8 \mathrm{~mL} / \mathrm{min}$ with a temperature maintained at $30^{\circ} \mathrm{C}$. The UV-vis peaks' spectra were recorded ranging from 200 to $650 \mathrm{~nm}$.

MS analyses were performed by using a Thermo Finnigan (MA, USA) LCQ Advantage system MS spectrometer with an electrospray ionisation source and an 'Ion Trap' mass analyser. The MS spectra

Table 2

Conversion of polyunsaturated alcohols $\mathbf{2}$ to corresponding acids

\begin{tabular}{lcc}
\hline Substrate & Conversion time $(\mathrm{h})$ & Acid yields $(\%)$ \\
\hline $\mathbf{2 a}$ & 2 & 100 \\
$\mathbf{2 b}$ & 2 & 100 \\
$\mathbf{2 c}$ & 44 & 82 \\
$\mathbf{2 d}$ & 24 & 25 \\
\hline
\end{tabular}

a The reported data concern the maximum acid formation; extending the conversion time degradation phenomena begin. 


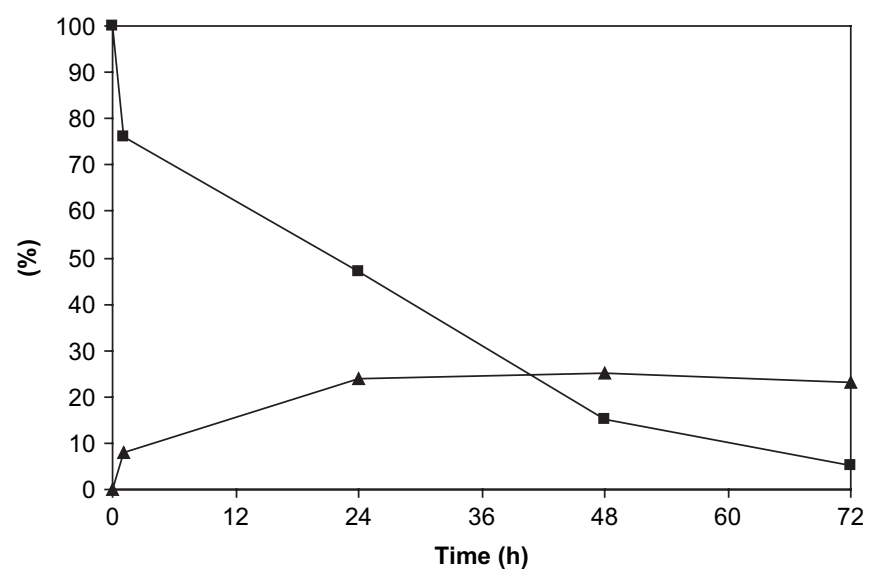

Figure 2. Time-course of the biotransformation of 2,4,6,8,10-dodecapentaenol 2d catalysed by A. aceti MIM2000/28. $\mathbf{\square}$, alcohol; $\boldsymbol{\Lambda}$, acid.

were obtained by direct infusion of a sample solution in a mixture $\mathrm{MeOH} / \mathrm{H}_{2} \mathrm{O} / \mathrm{AcOH}$ 10:89:1 under ionisation, ESI positive.

FTIR spectra were collected by using a Perkin-Elmer (MA, USA) FT-IR Spectrometer 'Spectrum One' in a spectral region between 4000 and $450 \mathrm{~cm}^{-1}$. Samples were mixed in a mortar with $\mathrm{KBr}$ (1:100) and pressed in a hydraulic press (14 tons) to small tablets, which were then analysed by transmittance technique with 32 scansions and $4 \mathrm{~cm}^{-1}$ resolutions.

All reagents, solvents and spectroscopic solvent grade employed for HPLC and MS analyses and DMSO- $d_{6}$ for NMR were purchased from Aldrich Chemical.

\subsection{General procedure to obtain alcohols $2 a-f$}

To a stirred solution of aldehyde 1a-f $(2.2 \mathrm{mmol})$ in absolute ethanol $(20 \mathrm{~mL}), \mathrm{NaBH}_{4}(3.3 \mathrm{mmol})$ in absolute ethanol $(5 \mathrm{~mL})$ was added under nitrogen. The reaction mixture was stirred at room temperature for $1-5 \mathrm{~h}$ (monitored by TLC). After distilled water $(30 \mathrm{~mL})$ addition, the aqueous layer was extracted with diethyl ether $(3 \times 30 \mathrm{~mL})$ and the organic layers were dried under sodium sulfate, filtered and then evaporated under reduced pressure leading to a residue, which was purified by recrystallisation. Low solubility of compounds $2 \mathbf{e}$ and $2 \mathbf{2 f}$ did not allow ${ }^{13} \mathrm{C}$ analysis.

\subsubsection{Compound $2 \boldsymbol{a}^{23}$}

Reaction time: 1 h. Colourless oil; yield 90\%.

\subsubsection{Compound $\mathbf{2 b}^{24}$}

Reaction time: $1.5 \mathrm{~h}$. Recrystallisation from petroleum ether gave a white solid; yield $90 \%$; mp $99-100{ }^{\circ} \mathrm{C}$.

\subsubsection{Compound $2 c^{25}$}

Reaction time: $2 \mathrm{~h}$. Recrystallisation from ethanol; white solid; yield $70 \%$; mp $99-100{ }^{\circ} \mathrm{C}$.

\subsubsection{Compound $\mathbf{2} \boldsymbol{d}^{26}$}

Reaction time: $3 \mathrm{~h}$. Recrystallisation from ethyl acetate; pale yellow solid; yield $80 \%$; mp $203-204{ }^{\circ} \mathrm{C}$; $\lambda_{\mathrm{MeOH}}$ : 310.5, 324.1, $340.5 \mathrm{~nm} ; \nu_{\max }(\mathrm{KBr}): 3280,3186,3014,2920,2870,1684,1654$, $1378,1004,964 \mathrm{~cm}^{-1} ; \delta_{\mathrm{H}}\left(200 \mathrm{MHz}, \mathrm{CDCl}_{3}\right): 6.27-6.12(8 \mathrm{H}, \mathrm{m}, \mathrm{CH})$, 5.85-5.74 (2H, m, CHCH $\left.\mathrm{CH}_{2} \mathrm{CHCH}_{3}\right), 4.25\left(2 \mathrm{H}, \mathrm{d}, \mathrm{J} 5.8 \mathrm{~Hz}, \mathrm{CH}_{2} \mathrm{OH}\right)$, $1.82\left(3 \mathrm{H}, \mathrm{d}, J 6.6 \mathrm{~Hz}, \mathrm{CH}_{3}\right), 1.31\left(1 \mathrm{H}, \mathrm{s}, \mathrm{CH}_{2} \mathrm{OH}\right.$ exch. with $\left.\mathrm{D}_{2} \mathrm{O}\right) ; \delta_{\mathrm{C}}$ $\left(53 \mathrm{MHz}_{\mathrm{CDCl}}\right.$ ): 133.5, 133.1, 133.9, 131.8-130.3, 18.3; $\mathrm{MS}: \mathrm{MH}^{+}$, found 177. $\mathrm{C}_{12} \mathrm{H}_{16} \mathrm{O}$ requires 176 .

\subsubsection{Compound $2 \boldsymbol{e}$}

Reaction time: $5 \mathrm{~h}$. Recrystallisation from ethyl acetate; yellow solid; yield $60 \%$; $\mathrm{mp}$ dec $>200{ }^{\circ} \mathrm{C}$; $\lambda_{\mathrm{MeOH}}$ : 333.7, 350.5, $369.7 \mathrm{~nm}$; $\nu_{\max }(\mathrm{KBr}): 3422,2962,2924,2852,1654,1636,1004,964 \mathrm{~cm}^{-1} ; \delta_{\mathrm{H}}$ (200 MHz, $\left.\mathrm{CDCl}_{3}\right)$ : 6.27-6.12 (10H, m, CH), 5.78-5.73 (2H, m, $\left.\mathrm{CHCH}_{2} \mathrm{OH}, \mathrm{CHCH}_{3}\right), 4.03\left(2 \mathrm{H}, \mathrm{d}, J 7.3 \mathrm{~Hz}, \mathrm{CH}_{2} \mathrm{OH}\right), 1.76(3 \mathrm{H}, \mathrm{d}, J$ $\left.6.6 \mathrm{~Hz}, \mathrm{CH}_{3}\right), 1.35\left(1 \mathrm{H}, \mathrm{s}, \mathrm{OH}\right.$ exch. with $\left.\mathrm{D}_{2} \mathrm{O}\right)$; $\mathrm{MS}: \mathrm{MH}^{+}$, found 203. $\mathrm{C}_{14} \mathrm{H}_{18} \mathrm{O}$ requires 202.

\subsubsection{Compound $\mathbf{2 f}$}

Reaction time: $5 \mathrm{~h}$. Recrystallisation from ethyl acetate; dark yellow solid; yield $40 \%$; $\mathrm{mp}$ dec $>200{ }^{\circ} \mathrm{C}$; $\lambda_{\mathrm{MeOH}}$ : 354.9, 373.3, $394.9 \mathrm{~nm}$; $\nu_{\max }(\mathrm{KBr}): 3430,2962,2924,2852,1684,1654,1268$, $1098,1018,800 \mathrm{~cm}^{-1} ; \delta_{\mathrm{H}}\left(200 \mathrm{MHz}, \mathrm{CDCl}_{3}\right): 6.32-6.17(12 \mathrm{H}, \mathrm{m}, \mathrm{CH})$, 5.86-5.75 (2H, m, CHCH $\left.2 \mathrm{OH}, \mathrm{CHCH}_{3}\right), 4.02\left(2 \mathrm{H}, \mathrm{d}, \mathrm{J} 5.2 \mathrm{~Hz}, \mathrm{CH}_{2} \mathrm{OH}\right)$, $1.76\left(3 \mathrm{H}, \mathrm{d}, J 6.1 \mathrm{~Hz}, \mathrm{CH}_{3}\right), 1.31$ (s, $1 \mathrm{H}, \mathrm{OH}$ exch. with $\mathrm{D}_{2} \mathrm{O}$ ); $\mathrm{MS}: \mathrm{MH}^{+}$, found 229. $\mathrm{C}_{16} \mathrm{H}_{20} \mathrm{O}$ requires 228 .

\subsection{General procedure to obtain acids $3 a-f$}

Triethyl phosphonoacetate $(4.52 \mathrm{mmol}, 0.89 \mathrm{~mL})$ was added under nitrogen to a stirred suspension of sodium hydride (4.82 mmol, $192.85 \mathrm{mg}$ of a $1: 1 \mathrm{w} / \mathrm{w}$ dispersion in mineral oil) in anhydrous $\mathrm{THF}(20 \mathrm{~mL})$ at $0{ }^{\circ} \mathrm{C}$. By maintaining this temperature the appropriate aldehyde $(4.098 \mathrm{mmol})$ was added and the reaction mixture was allowed to stand overnight at $4{ }^{\circ} \mathrm{C}$. The mixture was then poured into ice water and the reaction product was extracted with diethyl ethers or precipitated in the reaction medium, filtered and washed with water. The ethyl esters were suspended in $5 \% \mathrm{KOH}$ in methanol $(50 \mathrm{~mL})$ and refluxed for about $30 \mathrm{~min}$. After cooling, the precipitate was filtered and washed with cold methanol. A solution of $5 \%$ aqueous $\mathrm{HCl}$ was added to the potassium salt until acidic $\mathrm{pH}$ was reached. Acids $\mathbf{3 a}-\mathbf{f}$ were filtered and washed with a little amount of water.

\subsubsection{Compound $3 \boldsymbol{a}^{27}$}

White solid; yield $23 \%$; mp $125-126{ }^{\circ} \mathrm{C}$.

\subsubsection{Compound $3 \boldsymbol{b}^{27}$}

Pale yellow solid; yield $23 \%$; mp $188-189{ }^{\circ} \mathrm{C} ; \lambda_{\mathrm{MeOH}}: 297 \mathrm{~nm}$; $\nu_{\max }(\mathrm{KBr}): 3017,2921,1682,1637,1611,1419,1375,1300,1154,1002$, 967, $922 \mathrm{~cm}^{-1}$; $\delta_{\mathrm{H}}(200 \mathrm{MHz}, \mathrm{DMSO}): 12.1(1 \mathrm{H}, \mathrm{s}, \mathrm{CHCOOH}$ exch. with $\left.\mathrm{D}_{2} \mathrm{O}\right), 7.15(1 \mathrm{H}, \mathrm{dd}, J 15.27,10.99, \mathrm{CH}=\mathrm{CHCOOH}), 6.7(1 \mathrm{H}, \mathrm{dd}, J$ 14.97, $10.69 \mathrm{~Hz}, \mathrm{CH}=\mathrm{CHCH}=\mathrm{CHCOOH}), 6.3-6.0(2 \mathrm{H}, \mathrm{m}, \mathrm{CH}), 5.9$ $\left(1 \mathrm{H}, \mathrm{dq}, J 6.72,13.13 \mathrm{~Hz}, \mathrm{CHCH}_{3}\right), 5.8(1 \mathrm{H}, \mathrm{d}, J 15.27 \mathrm{~Hz},=\mathrm{CHCOOH})$, 1.7 (3H, d, J $6.72 \mathrm{~Hz}, \mathrm{CH}_{3}$ ); $\delta_{\mathrm{C}}(53 \mathrm{MHz}, \mathrm{DMSO}): 168.3,145.1,141.4$, 135.5, 132.0, 128.4, 121.6, 19.0; MS: $\mathrm{MH}^{+}$, found $139 . \mathrm{C}_{8} \mathrm{H}_{10} \mathrm{O}_{2}$ requires 138.

\subsubsection{Compound $3 c^{28}$}

Yellow solid; yield $51 \%$; mp $258-260{ }^{\circ} \mathrm{C} ; \lambda_{\mathrm{MeOH}}: 329 \mathrm{~nm} ; \nu_{\max }$ (KBr): 3015, 2918, 2849, 1682, 1618, 1594, 1422, 1384, 1309, 1276, $1008,923 \mathrm{~cm}^{-1} ; \delta_{\mathrm{H}}(200 \mathrm{MHz}$, DMSO): 12 (1H, s, CHCOOH exch. with $\left.\mathrm{D}_{2} \mathrm{O}\right), 7.2(1 \mathrm{H}$, dd, $J 15.27,11.3 \mathrm{~Hz}, \mathrm{CH}=\mathrm{CHCOOH}), 6.7(1 \mathrm{H}, \mathrm{dd}, J$ 14.66, $10.69 \mathrm{~Hz}, \mathrm{CH}=\mathrm{CHCH}=\mathrm{CHCOOH}), 6.3-6.0(4 \mathrm{H}, \mathrm{m}, \mathrm{CH}), 5.9$ $\left(1 \mathrm{H}, \mathrm{dq}, J_{9} 6.72,13.13 \mathrm{~Hz}, \mathrm{CHCH}_{3}\right), 5.8(1 \mathrm{H}, \mathrm{d}, J 15.27 \mathrm{~Hz},=\mathrm{CHCOOH})$, 1.8 (3H, d, J $6.72 \mathrm{~Hz}, \mathrm{CH}_{3}$ ); $\delta_{\mathrm{C}}(53 \mathrm{MHz}, \mathrm{DMSO}): 168.4,144.6,141.2$, 137.8, 133.4, 132.4, 130.4, 130.1, 122.2, 19.0; MS: $\mathrm{MH}^{+}$, found 165 . $\mathrm{C}_{10} \mathrm{H}_{12} \mathrm{O}_{2}$ requires 164 .

\subsubsection{Compound $3 \boldsymbol{d}^{21}$}

Dark yellow solid; yield 20\%; mp 260-262 ${ }^{\circ} \mathrm{C}$.

\subsubsection{Compound $3 \boldsymbol{e}^{29}$}

Dark orange solid; yield $10 \%$; mp dec $>200^{\circ} \mathrm{C}$; $\lambda_{\mathrm{MeOH}}: 379 \mathrm{~nm}$; $\nu_{\max }(\mathrm{KBr}): 3012,2924,2852,1712,1621,1589,1447,1384,1368$, 
$1300,1274,1248,1227,1138,1008,916 \mathrm{~cm}^{-1} ; \delta_{\mathrm{H}}(200 \mathrm{MHz}$, DMSO): $12\left(1 \mathrm{H}, \mathrm{s}, \mathrm{CHCOOH}\right.$ exch. with $\left.\mathrm{D}_{2} \mathrm{O}\right), 7.3(1 \mathrm{H}, \mathrm{dd}, J 15.03$, 11.36, $C H=\mathrm{CHCOOH}), \quad 6.6 \quad(1 \mathrm{H}, \quad \mathrm{dd}, J \quad 10.99, \quad 14.66 \mathrm{~Hz}$, $\mathrm{CH}=\mathrm{CHCH}=\mathrm{CHCOOH}), 6.3-6.0(8 \mathrm{H}, \mathrm{m}, \mathrm{CH}), 5.9(1 \mathrm{H}, \mathrm{d}, J 15.03 \mathrm{~Hz}$, $=\mathrm{CHCOOH}), 5.8\left(1 \mathrm{H}, \mathrm{dq}, J 6.96,14.15 \mathrm{~Hz}, C \mathrm{CHCH}_{3}\right), 1.8(3 \mathrm{H}, \mathrm{d}, J$ $\left.6.96 \mathrm{~Hz}, \mathrm{CH}_{3}\right) ; \delta_{\mathrm{C}}(53 \mathrm{MHz}, \mathrm{DMSO}): 167.8,144.9,141.2,137.8,136.2$, 135.3-130.5, 129.8, 120.0, 18.7; MS: $\mathrm{MH}^{+}$, found 217. $\mathrm{C}_{14} \mathrm{H}_{16} \mathrm{O}_{2}$ requires 216 .

\subsubsection{Compound $3 \boldsymbol{f}^{30}$}

Dark orange solid; yield $10 \%$; mp dec $>200{ }^{\circ} \mathrm{C}$; $\lambda_{\mathrm{MeOH}}$ : $397 \mathrm{~nm}$; $\nu_{\max }(\mathrm{KBr}): 3011,2925,2853,1709,1621,1434,1374,1304,1260$, 1238, 1137, $1007 \mathrm{~cm}^{-1} ; \delta_{\mathrm{H}}(200 \mathrm{MHz}, \mathrm{DMSO}): 12(1 \mathrm{H}, \mathrm{s}, \mathrm{CHCOOH}$ exch. with $\left.\mathrm{D}_{2} \mathrm{O}\right), 7.3(1 \mathrm{H}$, dd, $J 15.03,10.99 \mathrm{~Hz}, \mathrm{CH}=\mathrm{CHCOOH}), 6.8-$ $6.0(11 \mathrm{H}, \mathrm{m}, \mathrm{CH}), 5.8(1 \mathrm{H}, \mathrm{d}, \mathrm{J} 15.03 \mathrm{~Hz},=\mathrm{CHCOOH}), 5.95-5.7(1 \mathrm{H}, \mathrm{m}$, $\left.\mathrm{CHCH}_{3}\right), 1.8\left(3 \mathrm{H}, \mathrm{d}, J 6.59 \mathrm{~Hz}, \mathrm{CHCH}_{3}\right) ; \mathrm{MS}: \mathrm{MH}^{+}$, found 243 . $\mathrm{C}_{16} \mathrm{H}_{18} \mathrm{O}_{2}$ requires 242 .

\subsection{Microorganism}

A. aceti MIM2000/28 from our collection (MIM: Microbiologia Industriale Milano) was employed. Microorganism was routinely maintained on GYC solid medium (glucose $50 \mathrm{~g} / \mathrm{L}$, yeast extract $10 \mathrm{~g} / \mathrm{L}, \mathrm{CaCO}_{3} 30 \mathrm{~g} / \mathrm{L}$, agar $12 \mathrm{~g} / \mathrm{L}, \mathrm{pH} 6.3$, distilled water) at $28^{\circ} \mathrm{C}$. The strain grown on GYC slants for $24 \mathrm{~h}$ at $28^{\circ} \mathrm{C}$ was inoculated into Erlenmeyer flask containing $50 \mathrm{~mL}$ of GLY medium (glycerol $25 \mathrm{~g} / \mathrm{L}$, yeast extract $10 \mathrm{~g} / \mathrm{L}, \mathrm{pH} 5$, distilled water) on a reciprocal shaker (120 spm).

\subsection{Biotransformation}

According to substrate reactivity the culture rate of $A$. aceti MIM2000/28 employed was $200 \mathrm{~mL}$ for the biotransformation of compounds $\mathbf{a}$ and $\mathbf{b}$ and $600 \mathrm{~mL}$ for the other substrates.

The cultures were centrifuged ( $4000 \mathrm{rpm}$ for $10 \mathrm{~min}$ ) and suspended in $0.1 \mathrm{M}$ phosphate buffers $(100 \mathrm{~mL})$ at $\mathrm{pH}$ 6.8. Polyunsaturated compounds dissolved in ethanol were added to the biotransformation system to give $1 \mathrm{mg} / \mathrm{mL}$ of substrate concentration and $10 \%$ of solvent.
Samples $(250 \mu \mathrm{L})$ were taken at intervals, brought to $\mathrm{pH} 1$ by addiction of $5 \% \mathrm{HCl}$, extracted with an equal volume of ethyl acetate and analysed by TLC (80\% ACOEt/MeOH) and HPLC/DAD in the conditions as above described.

\section{Acknowledgements}

This work was supported by Fondo Interno Ricerca Scientifica e Tecnologica (FIRST), Università degli Studi di Milano.

\section{References and notes}

1. Bertelli, A.; Pini, E.; Stradi, R. Patent WO 03/95403 A1, 2003.

2. Pini, E.; Rossi, E.; Celentano, G.; Stradi, R. Org. Prep. Proced. Int. 2002, 34, 198.

3. Pini, E.; Nava, D.; Stradi, R. Org. Prep. Proced. Int. 2003, 36, 166.

4. Morelli, R.; Loscalzo, R.; Stradi, R.; Bertelli, A.; Falchi, M. Drugs Exp. Clin. Res. 2003, 29, 95

5. Pini, E.; Bertelli, A.; Stradi, R.; Falchi, M. Drugs Exp. Clin. Res. 2004, 30, 203.

6. Tagliazucchi, D.; Ghelardoni, D.; Maltinti, S.; Ronca, G.; Conte, A.; Pini, E. Pharmacologyonline 2006, 3, 765.

7. Kuhn, R.; Grundmann, C. Chem. Ber. 1937, 70, 1318.

8. Blout, E. R.; Fields, M. J. Am. Chem. Soc. 1948, 70, 180

9. Lee, D. G.; Brownridge, J. R. J. Am. Chem. Soc. 1973, 95, 3033.

10. Wiberg, K. B.; Deutsch, C. J. J. Am. Chem. Soc. 1973, 95, 3034.

11. Balkrishna, S. B.; Childers, W. E.; Pinnick, H. W. Tetrahedron 1981, 37, 2091.

12. Grieco, P. A.; Wang, C. L.; Burke, S. D. J. Chem. Soc., Chem. Commun. 1975, 537.

13. Jones, G. Org. React. 1967, 15, 204.

14. Black, T. H.; Zhang, Y. Synth. Commun. 1995, 25, 15.

15. Arnel, B.; Maruoka, K.; Yamamoto, H. Tetrahedron 1995, 51, 4011.

16. Molinari, F.; Gandolfi, R.; Villa, R.; Urban, E.; Kiener, A. Tetrahedron: Asymmetry 2003, 14, 2041.

17. Romano, A.; Gandolfi, R.; Nitti, P.; Rollini, M.; Molinari, F. J. Mol. Catal. B: Enzym 2002, 17, 235

18. Mitsukura, K.; Sato, Y.; Yoshida, T.; Nagasawa, T. Biotechnol. Lett. 2004, 26, 1643.

19. Holland, H. L. Organic Synthesis with Oxidative Enzyme; Wiley-VCH: Weinheim, 1992.

20. Asai, T. Acetic Acid Bacteria; University of Tokyo: Tokyo, 1968.

21. Souto, A. A.; Acuna, A. U.; Armat-Guerri, F. Tetrahedron Lett. 1994, 35, 5907.

22. Molinari, F. Curr. Org. Chem. 2006, 10, 1247.

23. Chuitan, S.; Hartwing, J. Angew. Chem. 2004, 43, 4794

24. Troast, D. M.; Porco, J. Org. Lett. 2002, 4, 991.

25. Steven, L. V.; Smith, S.; Woodward, P. Tetrahedron 2005, 70, 1154.

26. D’Amico, K.; Manos, C.; Christiensen, R. J. Am. Chem. Soc. 1980, 102, 1977.

27. Kuhn, R.; Grundmann, C. Chem. Ber. 1936, 69, 227.

28. Kuhn, R.; Hoffer, A. Chem. Ber. 1931, 64, 1977.

29. Kuhn, R.; Grundmann, C. Chem. Ber. 1937, 70, 1325.

30. Schmitt, J.; Obermeit, A. Justus Liebigs Ann. Chem. 1941, 547, 291. 